\title{
Valores-trabalho, preços de reprodução e renda da terra
}

\section{RODOLFO HOFFMANN*}

Labor values, reproduction prices and land rent. The paper analyses the determination of labor values and reproduction prices, including economic systems with joint production and systems with intensive land rent. The discussion is based on very simple numeric examples, avoiding that the mathematical complications hinder the comprehension of basic questions of Marxist economic theory.

Keywords: Labor values; surplus value; reproduction prices; joint production; land rent.

JEL Classification: B51.

\section{INTRODUÇÃO}

Este artigo analisa a determinação de valores-trabalho e preços em esquemas econômicos muito simples, com apenas duas linhas de produção ou atividades. A análise pode ser estendida para a economia com $n$ atividades, mas nesse caso seria necessário utilizar álgebra de matrizes. ${ }^{1}$ Optou-se por limitar a análise a situações artificialmente simples, que permitem discutir os principais problemas conceituais, sem necessidade de usar uma notação matemática menos usual.

O valor-trabalho é o tempo de trabalho socialmente necessário à produção de uma mercadoria. Por ser um "tempo de trabalho", considera-se que o valor-trabalho é, evidentemente, uma variável medida em escala cardinal. Isso não significa

\footnotetext{
* Professor do Instituto de Economia da Unicamp e da ESALQ-USP, com apoio do CNPq. E-mail: hoffmannr@usp.br. Submetido: 9/Abril/2012; Aprovado: 16/Outubro/2012.

${ }^{1}$ Uma apresentação didática do tema, usando álgebra de matrizes, pode ser encontrada em Hoffmann (2013).
} 
que seja fácil medir o valor-trabalho em uma situação concreta como, por exemplo, o valor-trabalho da produção brasileira de arroz.

A escala de medida que é associada a uma variável não é determinada pelas dificuldades práticas de mensuração. O PNB do Brasil é uma grandeza cardinal, mas seria ingenuidade acreditar que o valor laboriosamente obtido pelo IBGE seja exatamente verdadeiro. ${ }^{2}$

Não é trivial determinar o tempo "socialmente necessário" da definição do valor-trabalho de uma mercadoria. Mas nos esquemas analisados neste artigo admite-se que todos os trabalhadores são igualmente eficientes (trabalham com a mesma intensidade ${ }^{3}$ ), fazendo com que o tempo "socialmente necessário" coincida com o tempo efetivo de trabalho.

Os preços determinados nos esquemas apresentados adiante são apropriadamente denominados de "preços de reprodução" por Possas (1982, p. 89; 1984, p. 78). Nesses esquemas admite-se que a economia em um período reproduz exatamente o que ocorre no período anterior. ${ }^{4}$

Na próxima seção será analisado um esquema de produção simples de mercadorias, isto é, uma economia na qual todo o capital é circulante e não há produção conjunta (nem renda da terra). Cabe assinalar que nos esquemas sraffianos, o capital fixo e o uso da terra correspondem a casos especiais de produção conjunta. $\mathrm{Na}$ produção de milho, o trator novo usado como insumo aparece como "trator com um ano de uso" nos "produtos" do processo, e a área de terra aparece tanto entre os insumos como entre os "produtos". Assim, o produto é composto por milho, trator e terra.

É comum que artigos sobre a questão da transformação de valores-trabalho em preços se limitem à produção simples de mercadorias. É o caso, por exemplo, de Gontijo (2009). ${ }^{5}$ Não há dúvida de que a produção conjunta torna a análise mais complicada. Mas, como veremos adiante, também abre novos horizontes.

\footnotetext{
${ }^{2}$ Nesse sentido, parece absurdo afirmar, como faz Belluzo (1998, p.135), que “a mais-valia marxista é uma relação aberta no sentido em que exprime a força variável do capital em sugar trabalho vivo, e que assim é ilegítimo fixar quaisquer das magnitudes que a compõem”. O caráter enigmático dessa sentença (e de muitas outras sentenças do mesmo texto) torna difícil apontar o que está errado. $\mathrm{O}$ autor está afirmando que é ilegítimo atribuir valores numéricos à mais-valia? Ou é ilegítimo atribuir valores numéricos a magnitudes (quais?) que compõem a mais-valia? É óbvio que a teoria marxista do valor-trabalho não se restringe a uma análise da relação entre valores-trabalho e preços de reprodução, mas é óbvio, também, que variáveis cardinais podem (e devem, em certas circunstâncias) ser quantificadas.

${ }^{3}$ Note-se que essa pressuposição exclui a possibilidade de analisar conceitos relevantes, como o de mais-valia relativa.

${ }^{4}$ Não é difícil introduzir um pseudodinamismo, no qual a população e todas as grandezas econômicas globais crescem com a mesma taxa (permanecendo constantes as grandezas per capita). Ver Pasinetti, 1977).

${ }^{5}$ O exemplo numérico com cinco setores usado por Gontijo (2008 e 2009) é incoerente, pois o setor III é definido como o setor de produção da mercadoria-dinheiro (ouro) e afirma-se que a cesta de consumo por unidade de trabalho é constituída por 10 unidades do produto do setor III. É óbvio que os trabalhadores não "consomem" ouro.
} 
A determinação de preços e valores-trabalho em esquemas limitados à produção simples de mercadorias é considerada na segunda seção. Essa é a situação de análise mais fácil e mais conhecida, mas é uma etapa importante para preparar o leitor para as situações mais complicadas estudadas em seguida. $\mathrm{Na}$ terceira seção é analisado um esquema com produção conjunta, adotando-se o método de determinação dos valores-trabalho descrito em Morishima e Catephores (1980), usando programação linear. Trata-se de um método que está longe de ser universalmente aceito. ${ }^{6} \mathrm{Na}$ quarta seção é analisada a determinação dos valores-trabalho em uma economia com renda da terra, um tema que Marx deixou "inacabado" e que nem foi abordado no livro de Morishima e Catephores.

Em esquemas sem produção conjunta, é possível obter inicialmente os valores-trabalho e depois transformá-los em preços de reprodução. Verifica-se que quando há produção conjunta (o que inclui esquemas com renda da terra), os preços de reprodução são necessariamente calculados a partir dos coeficientes técnicos do esquema, não fazendo mais sentido falar de uma "transformação de valores em preços".

\section{UM ESQUEMA COM PRODUÇÃO SIMPLES DE DUAS MERCADORIAS}

\section{O esquema e a determinação dos valores-trabalho}

Consideremos uma economia com apenas dois produtos; um meio de produção e um bem de consumo.

São dados os seguintes valores:

a) Quantidade produzida do meio de produção: $X_{1}=12$.

b) Quantidade do meio de produção utilizada no próprio setor: $X_{11}=8$.

c) Quantidade do meio de produção utilizada na produção de bens de consumo: $X_{12}=4$.

d) Quantidade produzida do bem de consumo: $X_{2}=4$.

e) Trabalho (em homens-dia ou equivalentes-homem) empregado na produção de meios de produção: $\mathcal{L}_{1}=4$.

f) Trabalho empregado na produção do bem de consumo: $\mathcal{L}_{2}=8$.

\footnotetext{
${ }^{6}$ Há muitos procedimentos alternativos sem base lógica. Um exemplo é Saad (1980), que reproduz o procedimento errado de Marx para transformar valores em preços e o defende, argumentando que se trata de um "problema qualitativo"(p. 142) e que "o procedimento de Marx é adequado para a derivação do conceito de preço de produção"(p. 144), como se cálculos errados pudessem ajudar a formar conceitos corretos. Outro exemplo é o artigo de Kliman e McGlone (1999), no qual simplesmente se estabelece que valores-trabalho e preços podem ser "compreendidos igualmente como montantes de dinheiro e de tempo de trabalho"(p. 36). Para uma avaliação crítica erudita do procedimento denominado "sistema temporal único" (temporal single-system), ver Gontijo (2008). Ver, também, a discussão crítica de Lucas e Serrano (2011). Note-se que nenhum dos dois artigos trata da relação entre valores-trabalho e preços em sistemas com produção conjunta.
} 
Seja $\lambda_{1}$ o valor-trabalho por unidade do meio de produção e seja $\lambda_{2}$ o valor-trabalho por unidade do bem de consumo. Então, temos:

$$
\left\{\begin{array}{l}
\lambda_{1} X_{11}+\mathcal{L}_{1}=\lambda_{1} X_{1} \\
\lambda_{1} X_{12}+\mathcal{L}_{2}=\lambda_{2} X_{2}
\end{array}\right.
$$

No caso desse exemplo numérico, temos

$$
\left\{\begin{array}{l}
8 \lambda_{1}+4=12 \lambda_{1} \\
4 \lambda_{1}+8=4 \lambda_{2}
\end{array}\right.
$$

Da primeira equação obtemos $\lambda_{1}=1$. Substituindo esse resultado na segunda equação obtemos $\lambda_{2}=3$.

$\mathrm{Na}$ literatura marxista é usual a seguinte notação, $\operatorname{com} c, v, m$ e $W$ indicando, respectivamente, capital constante, capital variável, mais-valia e valor-trabalho total:

$$
\left\{\begin{array}{l}
c_{1}+v_{1}+m_{1}=W_{1} \\
c_{2}+v_{2}+m_{2}=W_{2}
\end{array}\right.
$$

Para esse exemplo numérico temos

$$
\begin{array}{lll}
c_{1}=8 & v_{1}+m_{1}=4 & W_{1}=12 \\
c_{2}=4 & v_{2}+m_{2}=8 & W_{2}=12
\end{array}
$$

Vamos admitir que a taxa de mais-valia é $\mu=\frac{m}{v}=\frac{3}{5}=0,6$.

Então, $\frac{m}{m+v}=\frac{\mu}{1+\mu}=\frac{3}{8}$ e $\frac{v}{m+v}=\frac{1}{1+\mu}=\frac{5}{8}$.

Dessa maneira, obtém-se a seguinte tabela de valores-trabalho totais nos dois setores:

Tabela 1: Valores-trabalho totais nos dois setores

\begin{tabular}{c|c|c|c|c}
\hline Setor & $c$ & $\boldsymbol{v}$ & $\boldsymbol{m}$ & $\boldsymbol{W}$ \\
\hline 1 & 8 & 2,5 & 1,5 & 12 \\
2 & 4 & 5 & 3 & 12 \\
\hline Total & 12 & 7,5 & 4,5 & 24 \\
\hline
\end{tabular}

Note-se que a composição orgânica do capital é maior no setor de produção de meios de produção: $\frac{c_{1}}{v_{1}}=\frac{8}{2,5}=3,2$ e $\frac{c_{2}}{v_{2}}=\frac{4}{5}=0,8$.

\section{O destino da produção}

No exemplo numérico apresentado, em cada período (ano) são consumidas 12 unidades do meio de produção. Como a produção também é igual a 12 unidades, trata-se de uma economia em situação de "reprodução simples". O produto líquido está totalmente na forma de bens de consumo.

Admitimos que a taxa de mais-valia é igual a 0,6 , isto é, $\frac{m}{v}=\frac{3}{5}$. Então $\frac{v}{m+v}=\frac{5}{8}$. Isso significa, nesse exemplo, que os trabalhadores recebem (e consomem) $\frac{5}{8}$ do 
produto líquido, que correspondem a 2,5 unidades físicas. As restantes 1,5 unidades serão consumidas pelos capitalistas.

A cada unidade de trabalho correspondem

$\frac{2,5}{12}=\frac{5}{24}$ unidades de bem de consumo

Os trabalhadores empregados no setor 1 recebem

$\frac{5}{24} \cdot 4=\frac{5}{6}$ unidades

e os trabalhadores empregados no setor 2 recebem

$\frac{5}{24} \cdot 8=\frac{10}{6}$ unidades do bem de consumo.

\section{Determinação dos preços de produção da maneira usada por Marx}

A relação entre mais-valia $(m)$ e valor-trabalho do capital total $(c+v)$ é usualmente denominada "taxa de lucro". Mas esse é um conceito distinto da taxa de lucro em valores monetários. É muito inconveniente usar o mesmo nome para duas grandezas conceitualmente distintas e que, em geral, são numericamente diferentes, como será mostrado adiante.

A expressão "taxa de lucro" será reservada para a relação definida em valores monetários (e será indicada por $\pi$ ). A relação $m /(c+v)$ será denominada "taxa de lucro em valor-trabalho" (e será indicada por $\psi$ ).

$\mathrm{Na}$ Tabela 1 verifica-se que a mais-valia total é 4,5 e o valor-trabalho de todo o capital empatado é $12+7,5=19,5$. Então a taxa de lucro em valor-trabalho é

$$
\psi=\frac{4,5}{19,5}=\frac{9}{39}=0,231
$$

Aplicando essa taxa nos dois setores, e levando em consideração a quantidade física produzida, obtemos os seguintes "preços de produção" (por unidade física):

$$
\begin{aligned}
& p_{1}^{*}=\frac{1}{12}(8+2,5)\left(1+\frac{9}{39}\right)=\frac{42}{39}=\frac{14}{13}=1,077 \\
& p_{2}^{*}=\frac{1}{4}(4+5)\left(1+\frac{9}{39}\right)=\frac{108}{39}=\frac{36}{13}=2,769
\end{aligned}
$$

Esses "preços de produção" estão errados, até porque as expressões usadas são dimensionalmente inconsistentes. No segundo membro temos tempo de trabalho por unidade física do produto e no primeiro membro pretende-se obter valores monetários por unidade do produto. Os preços de reprodução corretos serão determinados adiante. Curiosamente, pode-se mostrar que o procedimento de Marx é o primeiro passo de um processo iterativo que converge para os preços de reprodução corretos.

Verifica-se que $\frac{p_{2}^{*}}{p_{1}^{*}}=\frac{36}{14}=\frac{18}{7}=2,571$.

Notar que $p_{1}^{*}>\lambda_{1}$ e $p_{2}^{*}<\lambda_{2}$, de acordo com a ideia de que "os preços de pro- 
dução tendem a ser relativamente maiores, em comparação com os valores, nos setores onde a composição orgânica do capital é maior".?

\section{Determinação correta dos preços de reprodução adotando a primeira mercadoria como unidade monetária}

Seja $p_{1}$ o preço do meio de produção, seja $p_{2}$ o preço do bem de consumo, seja $\pi$ a taxa de lucro (em termos monetários) e seja $w$ o salário. Então, admitindo que a taxa de lucro é a mesma nos dois setores, temos

$$
\left\{\begin{array}{l}
\left(X_{11} p_{1}+\mathcal{L}_{1} w\right)(1+\pi)=X_{1} p_{1} \\
\left(X_{12} p_{1}+\mathcal{L}_{2} w\right)(1+\pi)=X_{2} p_{2}
\end{array}\right.
$$

Para o exemplo numérico apresentado, temos

$$
\begin{aligned}
& \left(8 p_{1}+4 w\right)(1+\pi)=12 p_{1} \\
& \left(4 p_{1}+8 w\right)(1+\pi)=4 p_{2}
\end{aligned}
$$

Até aqui temos duas equações com quatro incógnitas: $p_{1}, p_{2}, w$ e $\pi$.

Vimos, anteriormente, que a cada unidade de trabalho corresponde $\frac{5}{24}$ unidades do bem de consumo. Então

$$
w=\frac{5}{24} p_{2} \text { ou } p_{2}=\frac{24}{5} w
$$

Como o nível geral de preços é, nesse modelo, arbitrário (depende da definição da unidade monetária), fazemos

$$
p_{1}=1
$$

O conjunto de equações (1), (2), (3) e (4) constitui um sistema de quatro equações com quatro incógnitas.

Substituindo (3) e (4) em (1) e (2), obtemos

$$
\begin{aligned}
& (8+4 w)(1+\pi)=12 \\
& (4+8 w)(1+\pi)=\frac{96}{5} w
\end{aligned}
$$

Dividindo membro a membro segue-se que

$$
\frac{2+w}{1+2 w}=\frac{5}{8 w} \text { ou } 8 w^{2}+6 w-5=0
$$

A única solução positiva é $w=0,5$. Substituindo em (3) obtemos

$$
p_{2}=2,4
$$

\footnotetext{
${ }^{7}$ Uma afirmativa que nem sempre é verdadeira, conforme se pode inferir da discussão em Pasinetti (1977, p. 82-84). A relação entre preços de produção e valores-trabalho de uma mercadoria depende não só da composição orgânica na indústria que a produz, mas também da composição orgânica nas indústrias que produzem seus insumos, e assim por diante.
} 
Note que $\frac{\lambda_{2}}{\lambda_{1}}=3>\frac{p_{2}^{*}}{p_{1}^{*}}=2,571>\frac{p_{2}}{p_{1}}=2,4$.

Substituindo $w=0,5$ em (5) ou (6), obtemos $\pi=0,2$ ou $20 \%$. Note-se que essa taxa é diferente da taxa de lucro em valor-trabalho $(\psi=0,231) .{ }^{8}$

Verifica-se que o lucro total é igual a 3,6.

\section{Lucro total numericamente igual à mais-valia}

Dada a arbitrariedade na escolha de uma unidade monetária, é possível determinar preços de reprodução que tornem o lucro total numericamente igual à mais-valia total. Cabe ressaltar que isso é um exercício totalmente irrelevante, pois se trata de grandezas dimensionalmente distintas.

Vimos, na Tabela 1, que a mais-valia total é igual a 4,5. De (1) e (2) conclui-se que o lucro total é $\left(12 p_{1}+12 w\right) \pi$.

Se escolhermos uma unidade monetária que torne o lucro total numericamente igual à mais-valia total, temos

$$
\left(12 p_{1}+12 w\right) \pi=4,5
$$

Essa equação substitui (4). Dessa maneira o sistema de equações fica

$$
\left\{\begin{array}{l}
\left(8 p_{1}+4 w\right)(1+\pi)=12 p_{1} \\
\left(4 p_{1}+8 w\right)(1+\pi)=4 p_{2} \\
w=(5 / 24) p_{2} \\
\left(12 p_{1}+12 w\right) \pi=4,5
\end{array}\right.
$$

Resolvendo, obtemos $p_{1}=1,25, p_{2}=3, \pi=0,2$ e $w=5 / 8=0,625$.

Verifica-se que as relações $\frac{p_{2}}{p_{1}}=2,4, \frac{p_{1}}{w}=2 \mathrm{e} \frac{p_{2}}{w}=4,8$ permanecem as mesmas. Evidentemente a mudança na unidade monetária não pode afetar os preços relativos.

A Tabela 2 mostra os valores monetários dos componentes do capital e do lucro nos dois setores, considerando os preços obtidos nesta seção.

Tabela 2: Valores monetários dos componentes do capital, do lucro e da produção total.

\begin{tabular}{ccccccc}
\hline Setor & Capital constante & Capital variável & Lucro & Total \\
\hline 1 & 10 & 2,5 & 2,5 & 15 \\
2 & 5 & 5 & 2 & 12 \\
\hline Total & 15 & 7,5 & 4,5 & 27 \\
\hline
\end{tabular}

Comparando as Tabelas 1 e 2 verifica-se que, por construção, o valor do lucro total é numericamente igual à mais-valia $(4,5)$. Entretanto, o valor monetário de toda a produção (27) é diferente do valor-trabalho total (24).

\footnotetext{
${ }^{8}$ Verifica-se que a relação entre o preço de reprodução $(p)$ e o valor-trabalho por unidade $(\lambda)$ é igual a 1 no setor 1 e é igual a 0,8 no setor 2 . Isso é obviamente incompatível com a adoção de um valor único para essa relação, como é feito no procedimento denominado "sistema temporal único".
} 


\section{Reprodução ampliada}

Vimos que o exemplo numérico apresentado corresponde a uma economia em reprodução simples, pois o consumo de meios de produção é igual à sua produção (12 unidades). Mas a análise da relação entre valores-trabalho e preços de reprodução não depende dessa característica da economia. Se, por exemplo, multiplicarmos os coeficientes relativos à produção de meios de produção por 3/2 e multiplicarmos os coeficientes relativos à produção de bens de consumo por $3 / 4$, teremos:

$$
\begin{array}{llll}
X_{11}=12 & , & \mathcal{L}_{1}=6 & \\
X_{12}=3 & X_{1}=18 \\
\mathcal{L}_{2}=6 & , & X_{2}=3
\end{array}
$$

O trabalho total utilizado continua sendo igual a 12. Mas, agora, a produção de meios de produção (18 unidades) é maior do que o seu consumo anual (15 unidades), existindo condições para a reprodução ampliada. Uma vez que multiplicar uma equação por uma constante não altera a sua solução, os valores-trabalho por unidade continuam sendo $\lambda_{1}=1$ e $\lambda_{2}=3$. Analogamente, as equações (1) e (2) ficam multiplicadas por constantes, não se alterando a sua solução. Mantida a taxa de mais-valia e o volume de trabalho empregado, a mais-valia total continua sendo igual a 4,5 e, admitindo que os trabalhadores não poupem, seu consumo total continua sendo igual a 2,5 unidades do bem de consumo. Assim, apesar de termos agora uma economia com possibilidade de reprodução ampliada, os valores-trabalho por unidade e os preços continuam os mesmos. Note que valores-trabalho por unidade e preços dependem dos coeficientes técnicos, isto é, das relações entre quantidades dentro de cada indústria, ao passo que a possibilidade de reprodução ampliada é determinada pelo tamanho relativo entre indústrias.

\section{A relação entre taxa de lucro e taxa de mais-valia}

No exemplo a taxa de mais valia $(\mu=m / v)$ foi fixada em 0,6 , de maneira que os trabalhadores ficavam com

$$
\frac{v}{v+m}=\frac{1}{1+\mu}=\frac{5}{8}
$$

do produto líquido. Como o produto líquido é igual a 4 unidades do bem de consumo e são ocupadas 12 unidades de trabalho, a cada uma correspondem

$$
\frac{1}{12} \cdot \frac{1}{1+\mu} \cdot 4=\frac{1}{3(1+\mu)}=\frac{5}{24}
$$

unidades do bem de consumo, dando origem à equação (3). Se não fixarmos o valor da taxa de mais-valia, obtemos $w=\frac{1}{3(1+\mu)} p_{2}$ ou

$$
p_{2}=3(1+\mu) w
$$

Com $p_{1}=1$, de (1), (2) e (9) obtemos 


$$
\left\{\begin{array}{l}
(8+4 w)(1+\pi)=12 \\
(4+8 w)(1+\pi)=12(1+\mu) w
\end{array}\right.
$$

Explicitando $w$ da primeira equação e substituindo na segunda, após uma série de manipulações algébricas obtemos uma equação do $2^{\circ}$ grau em $\pi$ cuja solução é

$$
\pi=1+\mu \pm \sqrt{1+\mu+\mu^{2}}
$$

Como a expressão com sinal positivo antes do radical leva a um valor negativo de $w$, a única solução aceitável é

$$
\pi=1+\mu-\sqrt{1+\mu+\mu^{2}}
$$

A Figura 1 mostra a forma dessa função, ilustrando a afirmativa de que uma taxa de mais-valia positiva é condição necessária e suficiente para que se tenha uma taxa de lucro positiva, que é o "Teorema Marxista Fundamental “(ver Morishima e Catephores, 1980, p. 38, nota 15; e Morishima, 1973, p. 53).

Figura 1: Relação entre taxa de lucro e taxa de mais-valia

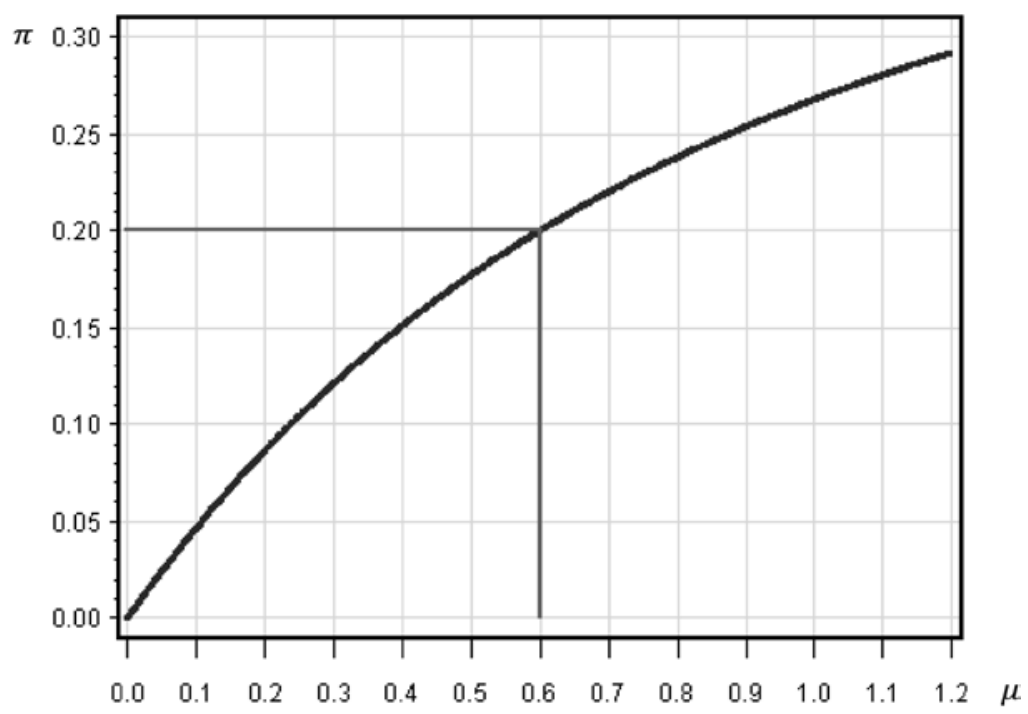

\section{PRODUÇÃO CONJUNTA}

\section{O sistema econômico e a determinação dos preços}

A determinação dos valores-trabalho e sua relação com os preços de reprodução se torna mais complicada quando há produção conjunta, isto é, o produto de uma indústria é composto por um conjunto de duas ou mais mercadorias. A Tabela 3 apresenta um exemplo numérico utilizado por Steedman (1977, p. 151), com apenas dois setores e duas mercadorias (A e B). 
Tabela 3: Fluxo de mercadorias em uma economia com dois setores (ou indústrias), ambos com produção conjunta

\begin{tabular}{c|c|c|c|c|c}
\hline \multirow{2}{*}{$\begin{array}{c}\text { Setor } \\
\text { (indústria) }\end{array}$} & \multicolumn{3}{|c|}{ Insumos } & \multicolumn{2}{c}{ Produto } \\
\cline { 2 - 6 } & Mercadoria A & Mercadoria B & Trabalho & Mercadoria A & Mercadoria B \\
\hline 1 & 25 & 0 & 5 & 30 & 5 \\
2 & 0 & 10 & 1 & 3 & 12 \\
\hline Total & 25 & 10 & 6 & 33 & 17 \\
\hline
\end{tabular}

Verifica-se que o produto líquido dessa economia é constituído por 8 unidades de A e 7 unidades de B. Admite-se que cada unidade de trabalho necessita, para sua manutenção e reprodução, de 0,5 unidade de $\mathrm{A}$ e $5 / 6$ unidade de $\mathrm{B}$, e que o salário é determinado por essa cesta de mercadorias. Sendo $p_{A}$ e $p_{B}$ os preços de A e B, respectivamente, temos

$$
w=0,5 p_{A}+\frac{5}{6} p_{B}
$$

Considerando os dados fornecidos na Tabela 3, devemos ter

$$
\begin{aligned}
& \left(25 p_{A}+5 w\right)(1+\pi)=30 p_{A}+5 p_{B} \\
& \left(10 p_{B}+w\right)(1+\pi)=3 p_{A}+12 p_{B}
\end{aligned}
$$

Fixando a unidade monetária de maneira que $p_{B}=1$, obtemos o seguinte sistema de três equações com três incógnitas (após simplificar a segunda equação):

$$
\left\{\begin{array}{l}
w=0,5 p_{A}+\frac{5}{6} \\
\left(5 p_{A}+w\right)(1+\pi)=6 p_{A}+1 \\
(10+w)(1+\pi)=3 p_{A}+12
\end{array}\right.
$$

Não é difícil verificar que a única solução satisfatória desse sistema é $p_{A}=0,161$, $w=0,914$ e $\pi=14,38 \%$.

\section{A determinação dos valores-trabalho}

De acordo com o procedimento usado anteriormente, os valores-trabalho $\lambda_{A}$ e $\lambda_{B}$ devem obedecer às equações

$$
\left\{\begin{array}{l}
25 \lambda_{A}+5=30 \lambda_{A}+5 \lambda_{B} \\
10 \lambda_{B}+1=3 \lambda_{A}+12 \lambda_{B}
\end{array}\right.
$$

$\mathrm{ou}$

$$
\left\{\begin{array}{c}
\lambda_{A}+\lambda_{B}=1 \\
3 \lambda_{A}+2 \lambda_{B}=1
\end{array}\right.
$$

Resolvendo, obtemos $\lambda_{A}=-1$ e $\lambda_{B}=2$. Steedman mostra, então, que a mais-valia seria negativa e que, de acordo com o exemplo, a taxa de lucro pode ser positiva mesmo com taxa de mais-valia negativa. Como bem argumentam Morishima e Catephores, o absurdo é admitir que o valor-trabalho possa ser negativo. O par de valores $\left(\lambda_{A}, \lambda_{B}\right)$ está, necessariamente, no primeiro quadrante, respeitando as 
desigualdades $\lambda_{A} \geq 0$ e $\lambda_{B} \geq 0$. Neste caso, os valores-trabalho não podem ser determinados por meio de um sistema de equações como (16) e é necessário recorrer à técnica de programação linear.

$\mathrm{Na}$ Tabela 3 verifica-se que cada unidade de trabalho empregada na indústria 2 gera um produto líquido de 3 unidades de $\mathrm{A}$ e 2 unidades de $\mathrm{B}$. Admitindo que os coeficientes técnicos não mudem com a escala de produção, se $\omega_{2}$ unidades de trabalho forem empregadas na indústria 2, obtém-se

$3 \omega_{2}$ unidades de $\mathrm{A}$ e $2 \omega_{2}$ unidades de $\mathrm{B}$.

Verifica-se, também, que na indústria 1 o produto líquido por unidade de trabalho é formado por 1 unidade de A e 1 unidade de B. Se essa indústria empregar $\omega_{1}$ unidades de trabalho, seu produto líquido será

$\omega_{1}$ unidades de A e $\omega_{1}$ unidades de B.

Então, empregando $\omega_{1}$ unidades de trabalho na indústria 1 e $\omega_{2}$ unidades de trabalho na indústria 2, o produto líquido obtido será

$\omega_{1}+3 \omega_{2}$ unidades de A e $\omega_{1}+2 \omega_{2}$ unidades de B.

Vamos admitir que se queira obter determinado produto líquido $Q$, constituído por $q_{\mathrm{A}}$ unidades de $\mathrm{A}$ e $q_{\mathrm{B}}$ unidades de $\mathrm{B}$, com o mínimo de esforço humano (trabalho). Trata-se de resolver o seguinte problema de programação linear:

$$
\text { Minimizar } \lambda_{Q}=\omega_{1}+\omega_{2}
$$

com

$$
\begin{aligned}
& \omega_{1}+3 \omega_{2} \geq q_{A} \\
& \omega_{1}+2 \omega_{2} \geq q_{B} \\
& \omega_{1} \geq 0 \text { e } \omega_{2} \geq 0
\end{aligned}
$$

O valor de $\lambda_{Q}$ na solução é o que Morishima e Catephores (1980) denominam o valor verdadeiro da mercadoria composta $Q$. Basta fazer $q_{1}=1$ e $q_{2}=0$ para obter, com o mesmo procedimento, o valor-trabalho por unidade de $A\left(\lambda_{A}\right)$. Obviamente, fazendo $q_{1}=0$ e $q_{2}=1$ obtemos o valor-trabalho por unidade de $B\left(\lambda_{B}\right)$.

Há um problema de programação linear que é o dual do problema descrito acima e cuja solução fornece os valores-sombra $\sigma_{A}$ e $\sigma_{B}$. O valor-sombra $\sigma_{A}$ é igual à redução que ocorre em $\lambda_{\mathrm{O}}$ se o valor de $q_{\mathrm{A}}$ na condição (18) for reduzido em 1 unidade, tornando-se menos limitante. Da mesma maneira, o valor-sombra $\sigma_{B}$ é igual à redução que ocorre em $\lambda_{Q}$ se o valor de $q_{\mathrm{B}}$ na restrição (19) for reduzido em uma unidade.

O teorema que estabelece a igualdade, no ponto ótimo, dos valores das funções objetivo do problema primal e do problema dual, nesse caso garante que

$$
\lambda_{Q}=\omega_{1}+\omega_{2}=\sigma_{A} q_{A}+\sigma_{B} q_{B}
$$

A Tabela 4 mostra o valor-trabalho de várias cestas de mercadorias $(Q$, com $q_{1}$ unidades de A e $q_{2}$ unidades de B) para o sistema de produção da Tabela 3. ${ }^{9}$

\footnotetext{
${ }^{9}$ Para obter uma tabela como essa é muito conveniente dispor de um programa de computador que resolva problemas como o caracterizado pelas equações (17) - (20), incluindo a solução do problema dual.
} 
O produto líquido do sistema inclui 8 unidades de $\mathrm{A}$ e 7 unidades de $\mathrm{B}$. $\mathrm{O}$ valor-trabalho dessa cesta é 3,5. Se a cesta tiver uma unidade a menos de B (ficando com 8 unidades de A e 6 unidades de B), o valor-trabalho cai para 3. Note-se que a redução no valor-trabalho $(3,5-3=0,5)$ é exatamente igual ao valor-sombra $\sigma_{B}$ quando se determina o valor-trabalho da cesta inicial.

Desnecessário dizer que a validade da expressão (21) pode ser verificada em qualquer linha da Tabela 4.

Os valores-trabalho por unidade para as mercadorias A e B são, respectivamente, $\lambda_{A}=0,333=1 / 3$ e $\lambda_{B}=0,5$.

Tabela 4: Valores-trabalho de cestas com $q_{\mathrm{A}}$ unidades da mercadoria $\mathrm{A}$ e $q_{\mathrm{B}}$ unidades da mercadoria B, para o sistema de produção descrito na Tabela 3

\begin{tabular}{|c|c|c|c|c|c|c|c|}
\hline \multirow{2}{*}{ Cesta } & \multirow[b]{2}{*}{$q_{\mathrm{A}}$} & \multirow[b]{2}{*}{$q_{\mathrm{B}}$} & \multirow{2}{*}{$\begin{array}{c}\text { Valor } \\
\lambda_{Q}\end{array}$} & \multicolumn{2}{|c|}{ Solução } & \multicolumn{2}{|c|}{ Valores-sombra } \\
\hline & & & & $\omega_{1}$ & $\omega_{2}$ & $\sigma_{A}$ & $\sigma_{B}$ \\
\hline Prod. total do sistema & 33 & 17 & 11 & 0 & 11 & 0,333 & 0 \\
\hline Produto líquido & 8 & 7 & 3,5 & 0 & 3,5 & 0 & 0,5 \\
\hline Produto líquido - 1 B & 8 & 6 & 3 & 0 & 3 & 0 & 0,5 \\
\hline Capital constante & 25 & 10 & 8,333 & 0 & 8,333 & 0,333 & 0 \\
\hline Capital variável & 3 & 5 & 2,5 & 0 & 2,5 & 0 & 0,5 \\
\hline Mais-valia & 5 & 2 & 1,667 & 0 & 1,667 & 0,333 & 0 \\
\hline 1 unidade de $A$ & 1 & 0 & 0,333 & 0 & 0,333 & 0,333 & 0 \\
\hline 1 unidade de $B$ & 0 & 1 & 0,5 & 0 & 0,5 & 0 & 0,5 \\
\hline Cesta-salário & 0,5 & $5 / 6$ & $5 / 12$ & 0 & $5 / 12$ & 0 & 0,5 \\
\hline
\end{tabular}

Calculando o valor do produto líquido com base nos valores-trabalho por unidade de A e B, obtemos

$$
8 \cdot \frac{1}{3}+7 \cdot 0,5=6,167
$$

Entretanto, esse cálculo não é válido, pois, quando há produção conjunta, os valores-trabalho deixam de ser aditivos. $\mathrm{O}$ verdadeiro valor-trabalho do produto líquido é 3,5. Para obter o produto líquido, a necessidade de produzir 8 unidades de A não é limitante (notar que $\sigma_{A}=0$ ). Em geral ${ }^{10}$

$$
\lambda_{Q} \leq \lambda_{A} q_{A}+\lambda_{B} q_{B}
$$

Em um esquema com produção simples, como o analisado anteriormente, somando o capital variável $(v)$ e a mais-valia $(m)$ obtinha-se o valor-trabalho do produto líquido. Isso não é mais necessariamente observado quando há produção

${ }^{10}$ Como mostram Morishoma e Catephores (1980). 
conjunta. Na Tabela 4 temos $v=2,5$ e $m=1,667$. Então $v+m=4,167$. Mas o valor-trabalho do produto líquido é 3,5.

\section{A medida do grau de exploração}

Conforme os resultados apresentados na Tabela 4, a taxa de mais-valia é

$$
\mu=\frac{m}{v}=\frac{1,667}{2,5}=0,667 \text { ou } 66,7 \%
$$

O trabalho direto total empregado é igual a 6 (ver Tabela 3). Então o trabalho excedente (não pago) é $6-2,5=3,5$ e a relação entre trabalho excedente e trabalho pago $(v)$ é

$$
\mu^{*}=\frac{6-v}{v}=\frac{3,5}{2,5}=1,4 \text { ou } 140 \%
$$

No caso da produção simples de mercadorias, como o examinado anteriormente, essas duas medidas do grau de exploração são sempre iguais $\left(\mu^{*}=\mu\right)$. Com produção conjunta tem-se $\mu^{*} \geq \mu$ e Morishima e Catephores (1980, p. 53) argumentam que $\mu^{*}$ "pode ser considerada como a medida completa de exploração", e que a taxa de mais-valia $(\mu)$ "representa uma medida imperfeita".

Note-se que o valor de $\mu^{*}$ também pode ser obtido utilizando o valor-trabalho da cesta de produtos que define o salário, apresentada no parágrafo antes de (12). Esse valor está na última linha da Tabela 4, e é igual a 5/12. Então,

$$
\frac{1-\frac{5}{12}}{\frac{5}{12}}=1,4=\mu^{*}
$$

\section{Exploração e taxa de lucro}

Para variar a taxa de exploração $\mu^{*}$ vamos alterar a cesta-salário, mas mantendo a relação entre seus componentes. A cesta-salário considerada até agora é igual a 0,5 unidades de A e 5/6 unidades de B. Introduzindo um coeficiente $\theta \geq 0$, passamos a considerar uma cesta-salário formada por $0,5 \theta$ unidades de A e $5 \theta / 6$ unidades de B. Em um problema de programação linear como o definido pelas equações $(17)-(20)$, mudanças proporcionais na cesta $\left(q_{\mathrm{A}}, q_{\mathrm{B}}\right)$ levam a mudanças na mesma proporção em $\lambda_{Q}$.

Então, lembrando (23), conclui-se que a taxa de exploração com uma cesta-

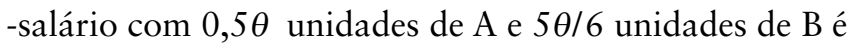

$$
\mu^{*}=\frac{1-\frac{5}{12} \theta}{\frac{5}{12} \theta}
$$

Segue-se que

$$
\begin{array}{r}
\mu^{*}=\frac{12-5 \theta}{5 \theta} \\
\text { e } \quad \theta=\frac{12}{5\left(\mu^{*}+1\right)}
\end{array}
$$


Lembrando (12), sabe-se que o salário é dado por

$$
w=\theta\left(0,5 p_{A}+\frac{5}{6} p_{B}\right)
$$

De (25) e (26), com $p_{B}=1$, obtemos

$$
w=\frac{6 p_{A}+10}{5\left(\mu^{*}+1\right)}
$$

Como as duas últimas equações do sistema (15) continuam válidas, temos o seguinte sistema:

$$
\left\{\begin{array}{l}
w=\frac{6 p_{A}+10}{5\left(\mu^{*}+1\right)} \\
\left(5 p_{A}+w\right)(1+\pi)=6 p_{A}+1 \\
(10+w)(1+\pi)=3 p_{A}+12
\end{array}\right.
$$

A partir desse sistema, definindo $\alpha=1+\mu^{*}$ e efetuando uma série de operações algébricas, é possível obter a equação

$$
(250 \alpha+110)(1+\pi)^{2}-(600 \alpha+96)(1+\pi)+345 \alpha=0
$$

Uma das raízes dessa equação do $2^{\circ}$ grau leva sempre a valores de $p_{\mathrm{A}}$ negativos, de maneira que a única raiz que produz valores aceitáveis é

$$
\begin{array}{rlrl}
\pi & =\frac{600 \alpha+96+\sqrt{\Delta}}{2(250 \alpha+110)} \\
\text { com } \quad \Delta & =(600 \alpha+96)^{2}-1380 \alpha(250 \alpha+110) \\
\mathrm{e} \quad & \alpha & =1+\mu^{*}
\end{array}
$$

Pode-se verificar que (29) fornece valores de $\pi$ que correspondem a valores positivos de $p_{\mathrm{A}}$ e $w$ se $\mu^{*}>1,2$. Como seria de esperar, a taxa de lucro $(\pi)$ é uma função crescente da taxa de exploração $\left(\mu^{*}\right)$, como ilustra a Figura 2.

Figura 2: A relação entre taxa de lucro e taxa de exploração para a economia esquematizada na Tabela 3

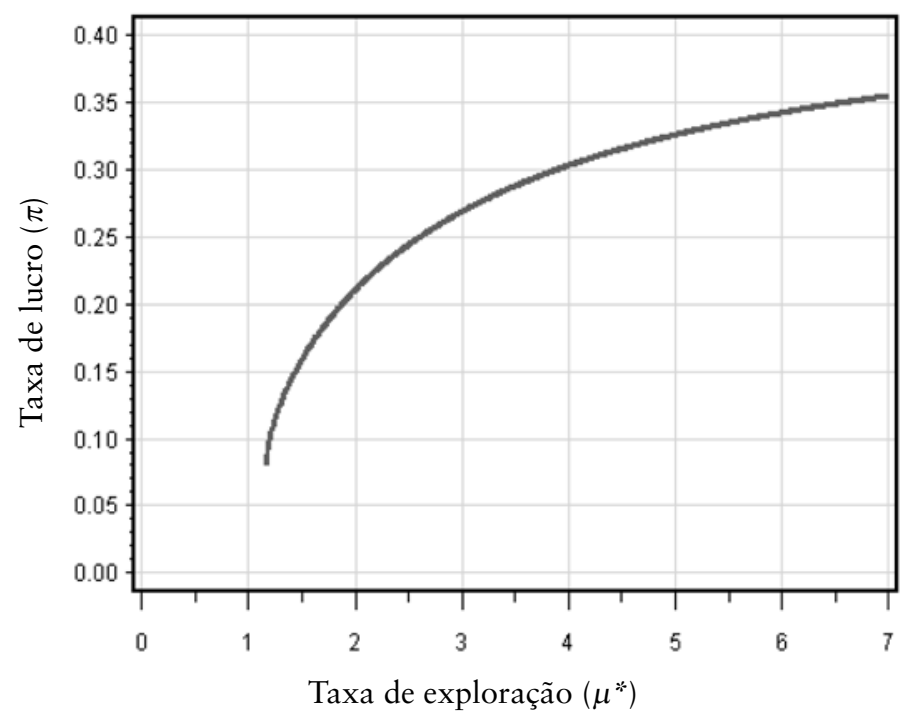




\section{RENDA DA TERRA}

\section{Um esquema com terra homogênea}

É bem conhecido que David Ricardo mostrou como o uso simultâneo de terras com qualidades diferentes (diferentes fertilidades, por exemplo) leva à existência de renda diferencial. Karl Marx assinala, corretamente, que pode haver renda mesmo na terra de pior qualidade que está sendo usada (a terra marginal), que ele denomina renda absoluta. Silva (1981) mostra que a explicação de Marx para a existência de renda absoluta é inapropriada, inclusive por depender da pressuposição de que a composição orgânica do capital empregado na agricultura seja inferior à composição orgânica média. Silva (1981) constrói um exemplo numérico de um sistema de produção no qual pode haver renda mesmo na terra de pior qualidade que está sendo usada (a terra tipo $\mathrm{D}$ ). $\mathrm{O}$ autor pressupõe que a demanda total pelo produto agrícola seja de 350 mil toneladas. No exemplo numérico, a produção pode crescer de 350 a 370 mil toneladas, aumentando o investimento nas terras já utilizadas (tipos de A a D), sem necessidade de utilizar a terra tipo E, de qualidade imediatamente inferior à terra tipo D. Mas, se a demanda total estiver entre 270 e 350 , parte da área de terra tipo D não será utilizada e, portanto, não haverá renda na terra marginal utilizada. Conclui-se que o exemplo mostra que pode haver renda na terra marginal, mas não que isso seja um fenômeno geral. Cabe ressaltar que Silva usa o procedimento errado de Marx para "transformar" valores-trabalho em preços de produção, confundindo a taxa de lucro em valor-trabalho $(\psi)$ com a taxa de lucro $(\pi)$ em valores monetários.

Dada a dificuldade de encontrar uma explicação clara para a existência da renda absoluta, vamos analisar, nesta seção, um esquema no qual se admite que a terra é perfeitamente homogênea, não podendo haver renda diferencial. A renda da terra existente nessas condições pode ser associada com a renda absoluta de Marx, embora a maneira de explicar a determinação do seu montante seja totalmente diferente da exposição de Marx. ${ }^{11}$

No esquema apresentado na Tabela 5, a "economia" produz uma única mercadoria agrícola, com duas técnicas de produção, em 2 unidades de terra homogênea. Note-se que o único produto é utilizado como bem de consumo e também como insumo na produção. ${ }^{12}$

Vamos admitir que o consumo dos trabalhadores por unidade de trabalho é igual a 13/9 de unidade do produto. Então, o consumo total dos trabalhadores é

$$
12 \cdot \frac{13}{9}=\frac{52}{3}=17,333
$$

\footnotetext{
11 Melhor denominá-la "renda intensiva”, pois, como mostra Sraffa (1960), ela está associada ao uso de técnicas progressivamente mais intensivas de exploração da terra.

12 Um exemplo numérico com renda em terra homogênea e com produção de duas mercadorias pode ser encontrado em Hoffmann e Cunha (2001). Um exemplo com renda diferencial é apresentado em Cunha e Hoffmann (2000).
} 
Tabela 5: Insumos e produção por unidade de área cultivada (em terra homogênea) para duas técnicas de produção de um único produto agrícola

\begin{tabular}{c|c|c|c|c}
\hline \multirow{2}{*}{ Técnica } & \multicolumn{3}{|c|}{ Insumos } & \multirow{2}{*}{ Produção } \\
\cline { 2 - 4 } & Produto & Trabalho & Terra & 22 \\
2 & 12 & 4 & 1 & 24 \\
\hline TOTAL $^{(1)}$ & 8 & 8 & 1 & 46 \\
\hline
\end{tabular}

(1) Esse total só existe se cada técnica for utilizada em 1 unidade de terra.

O produto líquido é formado por $46-20=26$ unidades do produto.

\section{Determinação dos preços e da taxa de lucro}

Sendo $p$ o preço do produto e sendo $\rho$ a renda por unidade de terra cultivada, com base nos dados da Tabela 5 podemos escrever as seguintes equações:

$$
\left\{\begin{array}{l}
(12 p+4 w)(1+\pi)+\rho=22 p \\
(8 p+8 w)(1+\pi)+\rho=24 p
\end{array}\right.
$$

Adotando uma unidade monetária tal que $p=1$, obtemos

$$
\left\{\begin{array}{l}
(12+4 w)(1+\pi)+\rho=22 \\
(8+8 w)(1+\pi)+\rho=24
\end{array}\right.
$$

Inicialmente vamos analisar esse sistema de duas equações com três incógnitas, sem fixar a cesta de consumo dos trabalhadores, isto é, permitindo que $w$ varie de zero até seu valor máximo.

Admite-se que há apenas 2 unidades de área da terra homogênea.

Se a demanda pelo produto pode ser atendida sem usar toda a terra disponível, a renda da terra será nula. Se fosse usada apenas a técnica 1 , a relação entre $w$ e $\pi$ seria obtida da equação (32):

$$
\left(12+4 w_{1}\right)(1+\pi)=22
$$

ou

$$
w_{1}=\frac{11}{2(1+\pi)}-3
$$

Se, também com terra excedente, fosse usada apenas a técnica 2, a relação entre $w$ e $\pi$ seria

$\left(8+8 w_{2}\right)(1+\pi)=24$

$\mathrm{ou}$

$$
w_{2}=\frac{3}{1+\pi}-1
$$

Se a demanda total pelo produto estiver entre 44 e 48, os dois métodos serão usados simultaneamente, não haverá terra excedente, $\rho>0$ e tanto a equação (32) como a (33) são válidas. Por diferença entre as duas, obtém-se

$$
\left(4 w_{3}-4\right)(1+\pi)=2
$$

ou

$$
w_{3}=\frac{1}{2(1+\pi)}+1
$$


Note-se que $w_{3}$ indica o salário obtido com o uso simultâneo das duas técnicas.

A figura 3 mostra como $w_{1}, w_{2}$ e $w_{3}$ variam em função de $\pi$. Verifica-se que as 3 curvas se cruzam no ponto em que $\pi=0,25$ e $w=1,4$.

Figura 3: As 3 funções $w-\pi$ para as duas técnicas descritas na tabela 5: uso exclusivo da técnica 1 (linha tracejada), uso exclusivo da técnica 2 (linha pontilhada) ou uso simultâneo das duas técnicas (linha contínua)

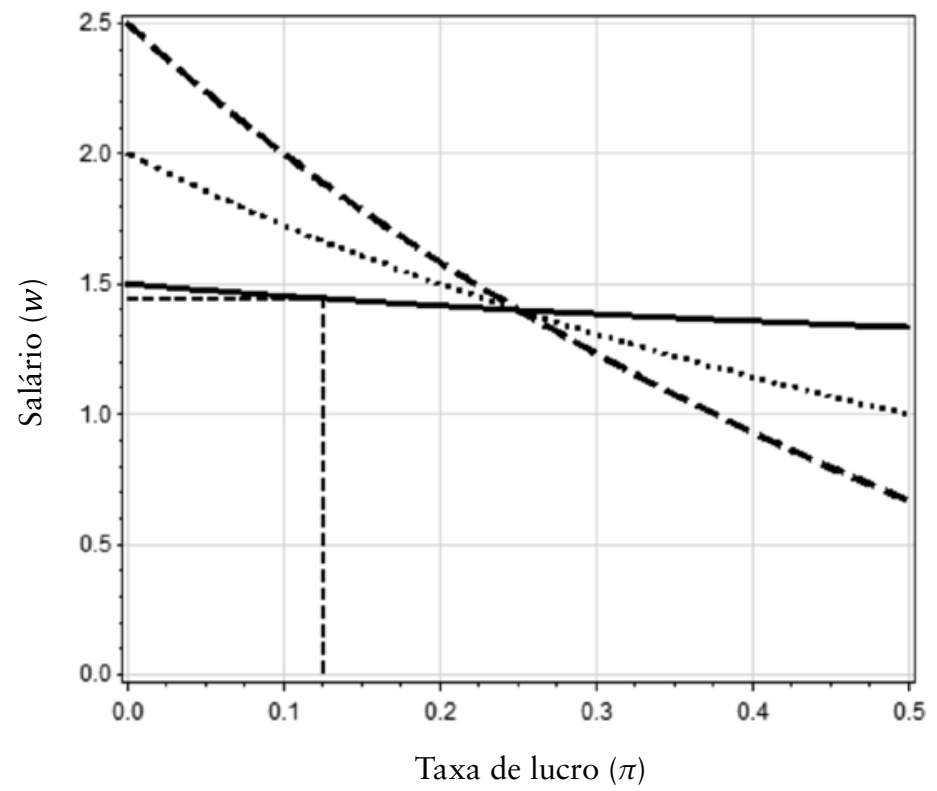

Observa-se, nessa figura, que para $\pi>0,25$ a técnica mais eficiente é a 2, pois, fixado o salário $(w)$, permite obter a maior taxa de lucro (também é a técnica que, fixado $\pi$, produz o maior valor de $w$ ). Como a técnica 2 é, também, a mais produtiva por unidade de área, será a única utilizada quando $\pi \geq 0,25, \operatorname{com} \rho=0$.

Para $\pi<0,25$ observa-se, na Figura 3, que a técnica 1 é mais eficiente que a técnica 2 . Se a demanda for inferior a 44 será utilizada apenas a técnica 1 , com $\rho=0$, pois nem toda a terra disponível será utilizada. Entretanto, se a demanda estiver entre 44 e 48, será necessário usar também a técnica 2, o salário vigente passa a ser $w_{3}$ e, substituindo (36) em (32) ou (33), obtém-se a seguinte equação para a renda da terra por unidade de área:

$$
\rho=4(1-2 \pi) \text { para } 0 \leq \pi \leq 0,25
$$

Conclui-se que, se a demanda estiver entre 44 e 48, a relação $w$ - $\pi$ para o esquema apresentado nas duas primeiras linhas da tabela 5 é formada pela função (36) (linha contínua na Figura 3) para $0 \leq \pi \leq 0,25$ e pela função (35) (linha pontilhada na Figura 3) para $0,25 \leq \pi \leq 2$.

Note-se que a técnica 1 seria usada isoladamente apenas quando $0 \leq \pi<0,25$ e a demanda pelo produto fosse inferior a 44 . 
É interessante notar que o fato de a terra ser ou não "escassa" depende da taxa de lucro, isto é, de um dos parâmetros que caracteriza a distribuição funcional da renda.

Vamos pressupor, agora, que a demanda está entre 44 e 48 e que a cesta-salário inclui apenas 13/9 de unidade do produto. Então, $\operatorname{com} p=1$,

$$
w=\frac{13}{9}=1,444
$$

De (36), (37) e (38) obtemos $\pi=0,125$ e $\rho=3$.

\section{Determinação dos valores-trabalho}

Note-se que com a técnica 1 obtém-se um produto líquido de 10 unidades da única mercadoria por unidade de área e com a técnica 2 o produto líquido é de 16 unidades da mercadoria por unidade de área.

Se $\omega_{1}$ e $\omega_{2}$ são, respectivamente, as áreas nas quais são usadas as técnicas 1 e 2 descritas na Tabela 5, define-se o valor-trabalho $\lambda_{Q}$ de uma cesta com $q$ unidades do produto por meio da solução do seguinte problema de programação linear:

$$
\begin{gathered}
\text { Minimizar } \lambda_{Q}=4 \omega_{1}+8 \omega_{2} \\
\text { com } \\
10 \omega_{1}+16 \omega_{2} \geq q \\
\omega_{1}+\omega_{2} \leq 2 \\
\omega_{1} \geq 0 \text { e } \omega_{2} \geq 0
\end{gathered}
$$

A restrição (41) refere-se à área de terra disponível.

A Tabela 6 mostra o valor-trabalho de diversas grandezas referentes ao sistema econômico representado na Tabela 5, com produção total 46 e produto líquido igual a 26. Para cada caso são apresentados os valores-sombra relativos às restrições (40) e (41), indicados, respectivamente, por $\sigma_{\mathrm{O}}$ e $\sigma_{S}$. Note-se que quando a restrição (41), relativa à área de terra disponível, é limitante, o valor de $\sigma_{S}$ é nega-

\begin{tabular}{|c|c|c|c|c|c|c|}
\hline \multirow[b]{2}{*}{ Cesta } & \multirow[b]{2}{*}{$q$} & \multirow[b]{2}{*}{ Valor $\lambda_{Q}$} & \multicolumn{2}{|c|}{ Solução } & \multicolumn{2}{|c|}{ Valores-sombra } \\
\hline & & & $\omega_{1}$ & $\omega_{2}$ & $\sigma_{Q}$ & $\sigma_{S}$ \\
\hline Produto líquido & 26 & 12 & 1 & 1 & $2 / 3$ & $-8 / 3$ \\
\hline Produto líquido - 1 & 25 & $34 / 3$ & $7 / 6$ & $5 / 6$ & $2 / 3$ & $-8 / 3$ \\
\hline Capital constante & 20 & 8 & 2 & 0 & 0,4 & 0 \\
\hline Capital variável & $52 / 3$ & $104 / 15$ & $26 / 15$ & 0 & 0,4 & 0 \\
\hline Mais-valia & $26 / 3$ & $52 / 15$ & $13 / 15$ & 0 & 0,4 & 0 \\
\hline 1 unidade & 1 & 0,4 & 0,1 & 0 & 0,4 & 0 \\
\hline Cesta-salário & $13 / 9$ & $21 / 45$ & $13 / 9$ & 0 & 0,4 & 0 \\
\hline
\end{tabular}
tivo, pois a desigualdade (41) tem sentido oposto ao da desigualdade (40).

Tabela 6: Valores-trabalho de q unidades da mercadoria única, para o sistema de produção descrito na Tabela 5 
De acordo com o teorema do dual, no ponto ótimo temos sempre

$$
\lambda_{Q}=4 \omega_{1}+\omega_{2}=q \sigma_{Q}+2 \sigma_{S}
$$

Para obter o produto líquido de 26 unidades, por exemplo, temos $\omega_{1}=\omega_{2}=1$, $\lambda_{Q}=12, \sigma_{Q}=2 / 3$ e $\sigma_{s}=-8 / 3$, verificando-se que

$$
26 \cdot \frac{2}{3}+2\left(-\frac{8}{3}\right)=\frac{36}{3}=12
$$

Sempre que a área de terra for limitante, haverá renda da terra $(\rho>0)$ e haverá um termo negativo no último membro de (43). Portanto, o valor-sombra negativo $\sigma_{s}$ corresponde, nessa "contabilidade" de valores-trabalho, à renda da terra determinada juntamente com os preços.

Da mesma maneira que já foi observado na Tabela 4, os valores-trabalho apresentados na Tabela 6 não são necessariamente aditivos. Somando o capital variável e a mais-valia obtemos

$$
\frac{104}{15}+\frac{52}{15}=\frac{156}{15}=10,4
$$

que é menor do que o valor-trabalho do produto líquido (12).

No final da seção referente ao exemplo com produção conjunta verificamos que $v+m$ superava o valor-trabalho do produto líquido. Agora, com renda da terra, a desigualdade se inverte, isto é, $v+m$ fica abaixo do valor-trabalho do produto líquido, uma situação que não foi prevista por Morishima e Catephores (1980).

Multiplicando o valor-trabalho de 1 unidade do produto $(0,4)$ por 26 obtemos 10,4, novamente menor do que o valor-trabalho do produto líquido. Para obter somente 1 unidade de produto líquido pode-se utilizar apenas a técnica com maior produtividade do trabalho, pois não há escassez de terra. Mas para obter 26 unidades de produto líquido em apenas 2 unidades de área, é necessário recorrer à técnica com maior produção por unidade de área à custa de menor produtividade do trabalho. Consequentemente, o tempo de trabalho necessário para obter as 26 unidades de produto líquido é maior do que 26 vezes o valor-trabalho de 1 unidade. ${ }^{13}$

Conforme os valores obtidos na Tabela 6, a taxa de mais-valia é

$$
\mu=\frac{\frac{52}{15}}{\frac{104}{15}}=0,5
$$

De acordo com o que foi discutido anteriormente, uma medida melhor do grau de exploração do trabalhador é a razão entre trabalho excedente (não pago) e trabalho pago:

$$
\mu^{*}=\frac{12-\frac{104}{15}}{\frac{104}{15}}=\frac{76}{104}=\frac{19}{26}=0,731
$$

\footnotetext{
${ }^{13} \mathrm{O}$ fato de os valores-trabalho não serem sempre aditivos não deveria causar estranheza. Embora se trate de conceito distinto, há uma analogia formal com um fenômeno conhecido por qualquer economista: o custo total cresce mais do que proporcionalmente à quantidade produzida quando a função de produção apresenta rendimentos decrescentes à escala.
} 
Nesse exemplo, a mesma medida pode ser obtida utilizando o valor-trabalho da cesta-salário:

$$
\mu^{*}=\frac{1-\frac{26}{45}}{\frac{26}{45}}=\frac{19}{26}
$$

\section{A relação entre a taxa de lucro e a taxa de exploração}

Para analisar como diversas características da economia variam em função da taxa de exploração, vamos introduzir um coeficiente $\theta$ que reduz ou aumenta a cesta-salário, da mesma maneira que foi feito na seção intitulada "Exploração e taxa de lucro". Em lugar de (38), temos:

$$
w=\frac{13 \theta}{9}
$$

Vimos que para $\theta=1$ obtemos $w=13 / 9=1,444$ e $\pi=0,125$. Para $\theta=63 / 65=$ 0,969231 obtemos $w=1,4$ e $\pi=0,25$. Para $\theta=27 / 26=1,03846$ obtemos $w=1,5$ e $\pi=0$. Como esperado, a relação $w-\pi$ é decrescente. ${ }^{14}$

Admitindo que os dados da Tabela 5 sejam os fluxos de insumos e produto de uma economia que dispõe de 2 unidades de área e emprega 12 unidades de trabalho, o valor do capital variável $(v)$ para cada valor de $\theta$ é obtido resolvendo o problema de programação linear definido pelas equações (39) - (42), com

$$
q=12 \cdot \frac{13 \theta}{9}=\frac{52}{3} \theta
$$

Em seguida, para cada valor de $\theta$, pode-se calcular a respectiva taxa de exploração, dada por

$$
\mu^{*}=\frac{12-v}{v}
$$

A Tabela 7 mostra os resultados obtidos para valores selecionados de $\theta$ e a Figura 4 ilustra a relação entre a taxa de lucro $(\pi)$ e a taxa de exploração $\mu^{*}$.

Tabela 7: Valores do capital variável $(\nu)$, da taxa de exploração $\left(\mu^{*}\right)$, da taxa de lucro $(\pi)$ e do salário $(W)$ para os valores selecionados do coeficiente $\theta$, considerando o esquema econômico da Tabela 5

\begin{tabular}{cccccc}
\hline$\theta$ & $52 \theta / 3$ & $v$ & $\mu^{*}$ & $\pi$ & $w$ \\
\hline 0,6 & 10,4 & 4,160 & 1,885 & 0,607 & 0,867 \\
0,9 & 15,6 & 6,240 & 0,923 & 0,304 & 1,300 \\
$63 / 65$ & 16,8 & 6,720 & 0,786 & 0,250 & 1,400 \\
1 & 17,333 & 6,933 & 0,731 & 0,125 & 1,444 \\
$27 / 26$ & 18,0 & 7,200 & 0,667 & 0 & 1,500 \\
1,05 & 18,2 & 7,280 & 0,648 & $(*)$ & - \\
\hline
\end{tabular}

Nota: Em nenhum dos casos considerados nesta tabela a restrição (41) é limitante, fazendo com que o respectivo valor-sombra seja nulo. O valor-sombra relativo à restrição (40) é sempre 0,4.

$\left({ }^{*}\right)$ A economia não é lucrativa.

\footnotetext{
${ }^{14}$ Mas isso nem sempre é verdadeiro em sistemas com renda da terra, como mostra o exemplo em Hoffmann e Cunha (2001).
} 
Figura 4: A taxa de lucro $(\pi)$ como função da taxa de exploração $\left(\mu^{*}\right)$ para o sistema econômico apresentado na Tabela 5

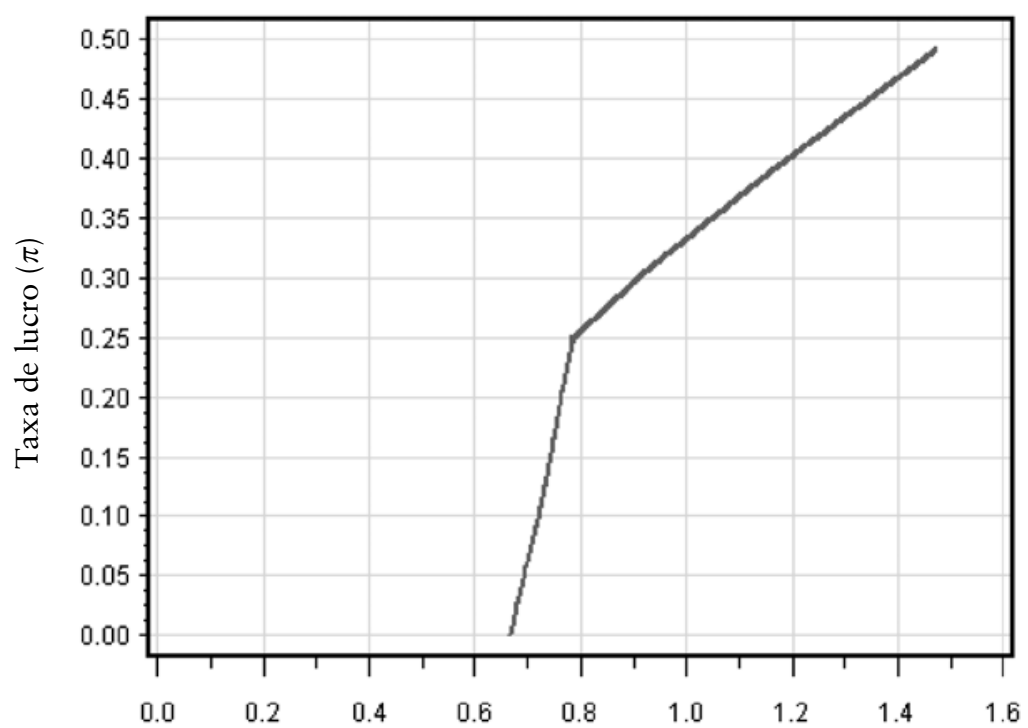

Taxa de exploração

Verifica-se, neste exemplo, que para $\theta \geq 27 / 26=1,03846$ há uma ampla faixa de valores positivos da taxa de exploração $\left(\theta<\mu^{*}<2 / 3\right)$ na qual a economia não é lucrativa. Conforme os resultados obtidos, quando as duas técnicas são usadas simultaneamente e $w=1,5$, têm-se $\pi=0$ e $\rho=4$, com o produto líquido (26) sendo esgotado com o pagamento dos salários $(12 \cdot 1,5=18)$ e da renda da terra $(2 \cdot 4=$ 8). Taxa de exploração positiva $\left(\mu^{*}>0\right)$ é uma condição necessária, mas não suficiente, para que haja taxa de lucro positiva $(\pi>0)$.

\section{CONSIDERAÇÕES FINAIS}

Embora Silva (1981) critique corretamente a explicação de Marx para a existência de renda absoluta, ele não se livra do uso do método incorreto de Marx na determinação dos preços de produção. Talvez seja isso que o induz a afirmar que o valor-trabalho de um produto agrícola é determinado pelo tempo de trabalho necessário à sua produção na terra de pior qualidade que esteja sendo utilizada. Dessa maneira, seu valor-trabalho total e sua mais-valia incluem um tempo de trabalho que nunca foi efetivamente executado. Conforme o conceito utilizado neste artigo, o valor-trabalho é a solução de um problema de programação linear que minimiza o tempo de trabalho socialmente necessário. Um tipo de terra ou uma técnica de cultivo com menor produto líquido por unidade de trabalho só entra no cômputo do valor-trabalho de certa cesta de mercadorias depois de esgotadas as 
possibilidades de uso das áreas de terra e/ou técnicas com maior produtividade do trabalho. Jamais se inclui no valor-trabalho de uma cesta de mercadorias um tempo de trabalho que não foi efetivamente realizado nas condições estabelecidas pelo esquema econômico considerado.

Ao determinar os preços de produção em um esquema onde há uso de terra (recurso natural) escassa, haverá um valor monetário positivo correspondente à renda da terra. Como a terra não tem, obviamente, valor-trabalho, não há, na "contabilidade" dos valores-trabalho, um elemento positivo correspondente à renda da terra. Mas um resultado interessante da análise desenvolvida neste artigo é que, ao calcular o valor-trabalho do produto líquido de um sistema econômico onde há escassez de terra aparece um termo negativo que representa o tempo de trabalho adicional que se tornou necessário devido à escassez de terra.

Nos exemplos numéricos apresentados neste artigo, tanto os preços de reprodução como os valores-trabalho foram sempre calculados a partir dos dados sobre fluxos físicos de insumos e produtos. Matematicamente, na ausência de produção conjunta, é possível determinar primeiro os valores-trabalho e depois "transformar" valores-trabalho em preços de reprodução, mas isso torna a análise mais complicada e não constitui, absolutamente, maneira de demonstrar a importância da teoria do valor-trabalho. ${ }^{15}$ Além disso, a obtenção dos preços e da taxa de lucro é indispensável para saber se determinado esquema pode efetivamente "funcionar" como uma economia capitalista lucrativa. Considere-se o exemplo da Tabela 5, com $w=1,6$. A determinação prévia dos valores-trabalho seria pura perda de tempo, pois é a análise dos preços (incluindo a determinação da renda de terra) que mostra que essa "economia" não é lucrativa.

Cabe explicitar que não é objetivo deste artigo defender ou criticar a importância que se dá à teoria do valor-trabalho. Procurou-se apenas deixar claro como valores-trabalho, preços de reprodução, mais-valia e taxa de lucro são grandezas que podem ser definidas, calculadas e relacionadas em esquemas econômicos com produção conjunta e com terra escassa.

\section{REFERÊNCIAS BIBLIOGRÁFICAS}

BELLUZZO, L. G. M. (1998). Valor e capitalismo: um ensaio sobre a Economia Política. 3. ed., Campinas, SP: UNICAMP-IE.

CUNHA, M. S.; HOFFMANN, R. (2000). Valores-trabalho e preços de produção em um sistema econômico sraffiano com renda extensiva. Revista de Economia Política, v. 20, n. 2 (78), abr.-jun. de 2000, p. 120-140.

GONTIJO, C. (2008). A transformação de valores em preços segundo o Sistema Temporal Único: uma apreciação crítica. Economia, v. 9, n. 1, jan./abr. de 2008, p. 215-243.

\footnotetext{
${ }^{15}$ Da mesma maneira que, na física, o fato de o conceito de massa ser fundamental para entender o que é o peso de um corpo, não significa que, para obter o peso de um corpo, seja "logicamente correto" determinar previamente sua massa. É muito mais fácil medir diretamente o seu peso.
} 
GONTIJO, C. (2009). O valor-trabalho como fundamento dos preços. Economia e Sociedade, v. 18, n. 3(37), dez. de 2009, p. 493-511.

HOFFMANN, R.; CUNHA, M. S. (2001). Valores-trabalho e preços de produção em sistemas econômicos sraffianos com terra homogênea. Revista Brasileira de Economia, v. 55, n. 1, jan.-mar. de 2001, p. 53-76.

HOFFMANN, R. (2013). Produtividade e preços em sistemas sraffianos. São Paulo, LP-Books.

KLIMAN, A J.; MCGLONE, T. (1999). A temporal single-system interpretation of Marx's value theory. Review of Political Economy, v. 11, n. 1, p. 33-59.

LUCAS, G.; SERRANO, F. (2011). A transformação dos preços em valores-trabalho: uma análise crítica das abordagens "monetaristas" para a teoria do valor de Marx. XVI Encontro Nacional de Economia Política, Uberlândia, 21 a 24 de junho de 2011.

MORISHIMA, M.; CATEPHORES, G. (1980). Valor, exploração e crescimento: Marx à luz da teoria econômica moderna. Rio de Janeiro, Zahar.

MORISHIMA, M. (1973). Marx's economics: a dual theory of value and growth. Cambridge University Press.

PASINETTI, L. L. (1977). Lectures on the theory of production. Columbia University Press.

POSSAS, M. L. (1982). Valor, preço e concorrência: não é preciso recomeçar tudo desde o início. Revista de Economia Política, v. 2, n. 4 (8), out.-dez. de 1982, p. 71-110.

POSSAS, M. L. (1984). Marx e os fundamentos da dinâmica econômica capitalista. Revista de Economia Política, v. 4, n. 3 (15), jul.-set. de 1984, p. 63-84.

SAAD FILHO, A. (2011). O valor de Marx: economia política para o capitalismo contemporâneo. Campinas, SP, Editora da Unicamp.

SILVA, S. S. (1981). Valor e renda da terra: o movimento do capital no campo. São Paulo, Editora Polis.

SRAFFA, P. (1960). Production of commodities by means of commodities. Prelude to a critique of economic theory. Cambridge University Press, reprinted 1972.

STEEDMAN, I. (1977). Marx after Sraffa. London, Unwin Brothers Limited. 